\title{
BIBLIOGRAPHY
}

1. R. E. Lane, Absolute convergence of continued fractions, Proc. Amer. Math. Soc. vol. 3 (1952) pp. 904-913.

2. R. E. Lane and H. S. Wall, Continued fractions with absolutely convergent even and odd parts, Trans. Amer. Math. Soc. vol. 67 (1949) pp. 368-380.

3. W. T. Scott and H. S. Wall, A convergence theorem for continued fractions, Trans. Amer. Math. Soc. vol. 47 (1940) pp. 155-172.

4. H. S. Wall, Analytic theory of continued fractions, New York, D. Van Nostrand Company, Inc., 1948.

The University OF TeXas AND

UNIVERSITY OF HOUSTON

\section{QUASI-BARRELLED LOCALLY CONVEX SPACES}

\section{MARK MAHOWALD AND GERALD GOULD}

1. Introduction and preliminary definitions. The main object of this paper is to answer some problems posed by Dieudonné in his paper Denumerability conditions in locally convex vector spaces [2]. His two main results are as follows:

Proposition 1. If $E$ is a barrelled space on which there is a countable fundamental system of convex compact subsets, [Definition 1.2] then it is the strong dual of a Fréchet-Montel Space.

PROPOSITION 2. If $E$ is either bornological or barrelled, and if there is a countable fundamental system of compact subsets, then $E$ is dense in the strong dual of a Frêchet-Montel Space.

Two questions raised by Dieudonné in connection with these results are:

(a) If $E$ is either bornological or barrelled then it is certainly quasibarrelled [1, Chapter 3, §2, Example 12]. Can one substitute this weaker condition on $E$ in Proposition 2?

(b) Is there is an example of a quasi-barrelled space which is neither barrelled nor bornological?

We shall show that the answer to (a) is "Yes," and that the answer to (b) is also "Yes," so that the generalization is in fact a real one.

A knowledge of the main results and notation of [1] is assumed. References to results occurring in the exercises there will however be given. These include in particular all the definitions and theorems

Presented to the Society January 29, 1960; received by the editors January 7, 1960. 
concerning bornological and quasi-barrelled (Infratonnelé) spaces. (For definitions and main properties, see [1, Chapter 3, §2, Examples $12,13,15,17]$.) The following definition explains the duality notation we shall use:

Definition 1.1. A vector space with a given locally convex topology will be denoted by the letter $E$ without a suffix. Various suffixes are used to denote the same point set with a related topology, as follows:

(a) $E_{\sigma}$ denotes the associated weak space (in the Bourbaki notation, the point set $E$ with the topologie affaiblie $\sigma\left(E, E^{\prime}\right)$ ).

(b) $E_{\tau}$ denotes the associated Mackey space, i.e., the point set $E$ with the Mackey topology $\tau\left(E, E^{\prime}\right)$.

(c) $E_{B}$ denotes the associated bornological space [1, Chapter 3, §2, Example 13].

(d) $E_{\omega}$ denotes the set $E$ with the finest locally convex topology. Various duals are defined as follows:

(e) $E^{\prime}$ denotes the topological dual of $E$ as a point set.

(f) $E^{\prime \sigma}$ denotes the weak dual of $E$.

(g) $E^{\prime \beta}$ denotes the strong dual of $E$.

(h) $E^{\prime k}$ denotes the set $E^{\prime}$ with the topology of uniform convergence over the compact sets of $E$.

(i) $E^{\prime c}$ denotes the set $E^{\prime}$ with the topology of uniform convergence over compact convex sets of $E$.

(j) $E^{\prime \tau}$ denotes the set $E^{\prime}$ with the Mackey topology.

(k) Corresponding to definitions (e), (f), (g), (h), (i), (j), will be topologies on the algebraic dual $E^{*}$; thus $E^{* k}$ will denote the space $E^{*}$ with the topology of uniform convergence over the compact sets of $E$.

(l) Completions will be denoted by: $\hat{E}, \hat{E}_{B}, \hat{E}_{\tau}, \hat{E}^{\prime \sigma}, \hat{E}^{\prime r}$, etc.

(m) If $E_{1}$ and $E_{2}$ denote the same point set, the statement $E_{1} \prec E_{2}$ means that the topology on $E_{1}$ is coarser than or equal to that on $E_{2}$.

As an illustration of this notation it should be noted that the following identities hold:

$$
E_{\sigma}=E^{\prime \sigma / \sigma}, \quad E_{\tau}=E^{\prime \sigma / c}, \quad E^{\prime \beta}={E_{\sigma}^{\prime \beta}}^{\beta}=E_{\tau}^{\prime \beta} .
$$

(The last equalities involve Mackey's theorems on boundedness and duality.)

The notation $\sigma(F, G)$ will be retained to denote weak topologies for spaces that are put $a$ priori in duality with each other.

The following further definitions are required:

Definition 1.2. Given a family $\mathfrak{S}$ of bounded sets of a topological 
vector space, then a sub-family $\mathfrak{F}$ is called a fundamental system of $\subseteq$ if each set of $\mathfrak{S}$ is contained in a set of $\mathfrak{F}$.

Definition 1.3. We say that a mapping from $E$ onto $F$ is almost open if the closure in $F$ of the image of a neighborhood of zero in $E$ is a neighborhood of zero in $F_{\tau}$. We say that $F^{\prime}$ is an almost closed subspace of $E^{\prime}$ if $U^{0} \cap F^{\prime}$ is closed in $E^{\prime}$ for every neighborhood $U$ of zero in $E$. Ptâk [5] showed that for locally convex spaces, continuous almost open mappings induce in the duals almost closed embeddings and conversely.

\section{A characterization of quasi-barrelled spaces.}

Lemma 2.1. Given a locally convex Hausdorff topological vector space $E$, then $E^{\prime \beta}$ is a topological subspace of $E_{B}^{\prime \beta}$.

Proof. The polars onto $E^{\prime}$ of the bounded sets $B$ (denoted by $\left.B^{01}\right)$ form a fundamental system of neighborhoods of zero in $E^{\prime \beta}$. Since the sets $B$ are also the bounded sets in $E_{B}$, the sets $B^{02}$ (polars of $B$ onto $E_{B}^{\prime}$ ) form a fundamental system of neighborhoods of zero in $E_{B}^{\prime \beta}$. The result now follows from the fact that $B^{01}=B^{02} \cap E^{\prime}$.

Lemma 2.2. If $E$ is quasi-barrelled, then $E=E_{\tau}$.

Proof. The bounded sets of $E$ and $E_{\tau}$ are coextensive so that $E^{\prime \beta}=E_{\tau}^{\prime \beta}$. Every compact convex set of $E_{\tau}^{\prime \beta}$ is equicontinuous ${ }^{1}$ and is therefore bounded in $E_{\tau}^{\prime \beta}$. Since $E$ is quasi-barrelled, every bounded set of $E^{\prime \beta}$ is equicontinuous [1, Chapter $3, \S 3$, Example 17]. Hence, every compact convex set of $E^{\prime \sigma}$ is equicontinuous; that is to say, $E=E_{\tau}$.

THEOREM 2.3. E is quasi-barrelled if and only if either of the following two equivalent conditions holds:

(a) the identity map from $E_{B}$ onto $E$ is almost open;

(b) $E^{\prime}$ is almost closed in $E_{B}^{\prime}$ and $E=E_{\tau}$.

Proof. In view of Ptâk's result (see Definition 1.3, together with Lemma 2.2) we shall merely prove that quasi-barrelledness of $E$ implies (b), and that (a) implies quasi-barrelledness. To show that condition (b) is necessary, it is sufficient to show (in view of the fact that $\left.E_{B}=E_{B \tau}\right)$ that if $K$ is a convex compact subset of $E_{B}^{\prime \sigma}$, then $K \cap E^{\prime}$ is a compact subset of $E_{B}^{\prime \beta}$. As an equicontinuous set of $E_{B}^{\prime}, K$ is bounded

1 It should be noted that although $E^{\prime \sigma}$ and $E_{\tau}^{\prime \sigma}$ refer to identical topological spaces, equicontinuous sets do not correspond. Thus $H$ is equicontinuous in $E_{\tau}^{\prime \sigma}$ if and only if $\bigcap_{u \in H} u^{-1}(V)$ is a neighborhood of zero in $E_{\tau}$, where $V$ is a given neighborhood of zero in the field of scalars on $E$. 
in $E_{B}^{\prime \beta}$. Hence from Lemma 2.1, $K \cap E^{\prime}$ is bounded in $E^{\prime \beta}$, and is therefore an equicontinuous subset of $E^{\prime}$ by virtue of the fact that $E$ is quasi-barrelled. It follows therefore that $K \cap E^{\prime}$ is relatively compact in $E^{\prime \sigma}$. Since, however, $E^{\prime \sigma}$ is a topological subspace of $E_{B}^{\prime \sigma}$, and $K$ is closed in $E_{B}^{\prime \sigma}, K \cap E^{\prime}$ is closed and therefore compact in $E^{\prime \sigma}$, and hence is a closed subset of $E_{B}^{\prime \sigma}$.

Finally, if condition (a) holds, and $U$ is a closed convex bornivorous set ${ }^{2}$ of $E$, it is the closure in $E$ of a bornivorous convex set of $E_{B}$; i.e., is the closure in $E$ of a neighborhood of zero for $E_{B}$, and hence by hypothesis, $U$ is a neighborhood of zero of $E_{\tau}$. It follows by definition that $E$ is quasi-barrelled.

It is worth noting that a similar theorem is available for barrelled spaces. Thus:

THEOREM 2.4. $E$ is barrelled if and only if $E=E_{\tau}$ and either of the following two equivalent conditions holds:

(a) the identity map from $E_{\omega}$ onto $E$ is almost open;

(b) $E^{\prime}$ is almost closed in $E^{* \sigma}$.

The proof is identical to that of Theorem 2.3 except for the following modifications. Every occurrence of " $E_{B}^{\prime \sigma}$ " or " $E_{B}^{\prime \beta}$ " is to be replaced by " $E^{* \sigma}$ "; every occurrence of " $E_{B}$ " is to be replaced by " $E_{\omega}$ "; every occurrence of " $E^{\prime \beta}$ " is to be replaced by " $E^{\prime \sigma}$ "; the word "quasibarrelled" is to be replaced everywhere by "barrelled," and finally, "bornivorous" is to be replaced on each occurrence by "absorbing."

3. Quasi-barrelled spaces with countability condition. We now have enough lemmas to prove the main result.

THEOREM 3.1. If E is quasi-barrelled and possesses a countable fundamental system of compact sets, then $E$ is dense in the strong dual of a Fréchet-Montel Space.

Proof. First of all it will be shown that $E^{\prime k}$ is a Fréchet Space. Clearly $E^{\prime k}$ is metric in view of the countability condition on $E$. It is sufficient therefore to deal with Cauchy sequences on $E^{\prime k}$. Let $\left\{x_{n}\right\}$ be such a Cauchy sequence, and let $x^{*}$ be its limit in $E^{* k}$. It is easy to show that the restriction of the functional $x^{*}$ onto a compact subset of $E$ is continuous; in particular, $x^{*}$ takes any convergent sequence in $E$ into a convergent sequence of scalars. A standard argument (cf. [1, Chapter 3, §2, Example 13]) now shows that $x^{*}$ takes bounded sets into bounded sets. Thus $x^{*} \in E_{B}^{\prime}[1$, Chapter 3, §2, Example 13],

${ }^{2} \mathrm{~A}$ set is bornivorous if it absorbs every bounded set. 
and hence $\hat{E}^{\prime k} \subset \hat{E}_{B}^{\prime}$. Since the set $\left\{x_{n}\right\} \cup\left\{x^{*}\right\}$ is compact in $\hat{E}^{\prime k}$, its closed convex hull $H$ will also be compact in $\hat{E}^{\prime k}$ and will therefore be compact as a subset of $E_{B}^{\prime \sigma}$. Hence from Theorem 2.3, $H \cap E^{\prime}$ is closed in $E_{B}^{\prime \sigma}$, and this implies that $x^{*}$ must be in $H \cap E^{\prime}$ along with $\left\{x_{n}\right\}$. Sequential completeness and therefore completeness of $E^{\prime k}$ now follows.

The rest of the proof largely uses the same techniques as in Dieudonné's work. The first step is to show that $E \subset E^{\prime k^{\prime}} \subset \hat{E}$. It is clear that $E \subset E^{\prime k^{\prime}}$ since $E^{\prime \sigma} \prec E^{\prime k}$. The inclusion is in general strict, since the convex hull of a relatively compact set of $E$ will not necessarily be a relatively compact set of $E$. On the other hand, a typical neighborhood $U$ of zero in $E^{\prime k}$ is the polar $K^{0}$ of a compact set $K$ of $E$. As a subset of $\hat{E}, K$ will be contained in a convex compact set of $\hat{E}$. It follows that $K^{0}$ is a neighborhood of zero in $\hat{E}^{\prime \tau}$, and hence that $E^{\prime k^{\prime}} \subset \hat{E}$. Denoting $E^{\prime k^{\prime}}$ with the topology induced from $\hat{E}$ by $E_{1}$, it follows from [1, Chapter IV, §2, Example 5], that the topologies in $E_{1}$ and $\hat{E}$ are identical respectively with $\tau\left(E_{1}, E^{\prime}\right)$ and $\tau\left(\hat{E}, E^{\prime}\right)$.

Next, we establish that $E^{\prime k^{\prime \prime}}=E^{\prime k^{\prime \prime} \tau}\left(=E_{1}\right)$. In fact, the completeness of $E^{\prime k}$ ensures that $E^{\prime k^{\prime k}}=E^{\prime k / c}$, and since a compact convex set of $E^{\prime k}$ is compact in the coarser topology $\sigma\left(E^{\prime k}, E_{1}\right)$, it follows that $E^{\prime k^{\prime \prime}} \prec E^{\prime k / \tau}$. On the other hand, if $K$ is a compact convex set in the topology $\sigma\left(E^{\prime}, E_{1}\right)$ it is also compact in $\sigma\left(E^{\prime}, E\right)$ and is therefore an equicontinuous subset of $E^{\prime}$; and as such, it is compact in $E^{\prime k}$ [1, Chapter III, §3, Proposition 5]. This establishes the reverse inequality $E^{\prime k / k}>E^{\prime k^{\prime} \tau}$, so that in fact $E^{\prime k^{\prime k}}=E^{\prime k^{\prime \prime} \tau}$, and this in turn implies $E_{1}$ is complete as the dual endowed with the compact open topology of the bornological space $E^{\prime k}$ [1, Chapter III, §3, Example 18]. Hence $E_{1}=\hat{E}$, since $E$ and therefore also $E_{1}$ is a dense subspace of $\hat{E}$.

Finally, $E_{1}$ is the strong dual of the Fréchet-Montel Space $E^{\prime k}$. The proof is as follows. The space $E_{1}$ is barrelled as the completion of a quasi-barrelled space. Since, however, $E_{1}$ is the Mackey dual of $E^{\prime k}$, bounded sets of $E^{\prime k}$ are relatively compact in the topology $\sigma\left(E^{\prime}, E_{1}\right)$, and as demonstrated in the preceding paragraph, such sets are relatively compact in $E^{\prime k}$. It follows therefore that $E_{1}=E^{\prime k^{\prime} \tau}=E^{\prime k^{\prime} \beta}$, and that $E$ is a Montel Space.

4. A counter example. The following construction yields an example of a quasi-barrelled space which is neither barrelled nor bornological.

Let $E_{1}$ be a barrelled, nonbornological space $[4 ; 6]$, and let $E_{2}$ be a bornological, nonbarrelled space. Since $E_{1}$ is not bornological, there exists a bornivorous set $B$ which is not a neighborhood of zero. Thus 
$B \times E_{2}$ is a bornivorous subset of $E_{1} \times E_{2}$ which is not a zero neighborhood for that space. Hence $E_{1} \times E_{2}$ is not bornological. In the same way, if $B$ is a barrel of $E_{2}$ which is not a neighborhood of zero, the subset $E_{1} \times B$ will be a barrel and yet fail to be a neighborhood in $E_{1} \times E_{2}$. Hence $E_{1} \times E_{2}$ is not barrelled. That $E_{1} \times E_{2}$ is quasi-barrelled follows from the following general result.

Theorem 4.1. If $\alpha$ is a cardinal for which it is true that the direct product of any $\alpha$ bornological spaces is bornological, then the product of any a quasi-barrelled spaces is quasi-barrelled.

Proof. Let $\left\{E_{i}\right\}, i \in I$, denote a family of quasi-barrelled spaces where $I$ has cardinality $\alpha$. Denoting the associated bornological space of each $E_{i}$ by $F_{i}$, the direct product $\prod E_{i}$ by $E$, and $E_{B}$ by $F$, we have that $F=\prod F_{i}$. This follows from the fact that $\prod F_{i}$ is (by hypothesis) bornological, and has the same system of bounded sets as $E$ (cf. [1, Chapter 3, \$2, Example 13]). From Theorem 2.3 the identity maps from $F_{i}$ and $E_{i}$ are almost open, and hence it is easy to see that the identity map from $F$ to $E$ is almost open and that $E=E_{\text {r. }}$ The result now follows from Theorem 2.3.

REMARK. It follows from [3, p. 337] that Theorem 4.1 is valid for any cardinal less than $\boldsymbol{\aleph}_{0}+2 \boldsymbol{\aleph}_{0}+2 \boldsymbol{\aleph}_{0}+\cdots$.

\section{REFERENCES}

1. N. Bourbaki, Espaces vectoriels topologiques, Chapters III-V, Paris, Hermann \& Cie, 1955.

2. J. Dieudonné, Denumerability conditions in locally convex vector spaces, Proc. Amer. Math. Soc. vol. 8 (1957) pp. 367-372.

3. W. Donoghue and K. Smith, On the symmetry and bounded closure of locally convex spaces, Trans. Amer. Math. Soc. vol. 73 (1952) pp. 321-344.

4. L. Nachbin, Topological vector spaces of continuous functions, Proc. Nat. Acad. Sci. U.S.A. vol. 40 (1954) pp. 471-474.

5. V. Ptak, Completeness and the open mapping theorem, Bull. Soc. Math. France vol. 86 (1958) pp. 41-74.

6. T. Shirota, On locally convex vector spaces of continuous functions, Proc. Japan Acad. vol. 30 (1954) pp. 294-299.

SyRACUSE UNIVERSITY 